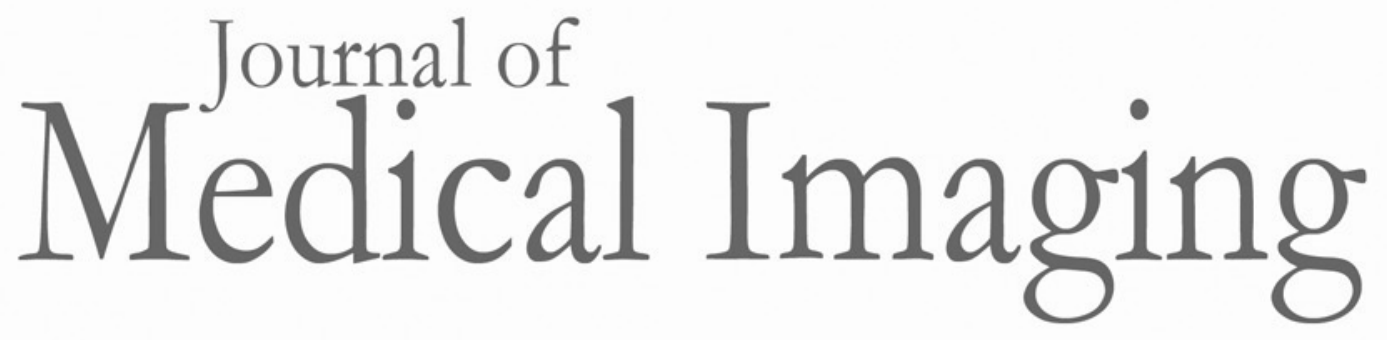

Medicallmaging.SPIEDigitalLibrary.org

\title{
Image-domain multimaterial decomposition for dual-energy computed tomography with nonconvex sparsity regularization
}

\author{
Qihui Lyu \\ Daniel O'Connor \\ Tianye Niu \\ Ke Sheng
}




\title{
Image-domain multimaterial decomposition for dual-energy computed tomography with nonconvex sparsity regularization
}

\author{
Qihui Lyu, ${ }^{\mathrm{a}}$ Daniel O'Connor, ${ }^{\mathrm{a}}$ Tianye Niu, ${ }^{\mathrm{b}, \mathrm{c}}$ and Ke Sheng, ${ }^{\mathrm{a}, *}$ \\ anniversity of California Los Angeles, Department of Radiation Oncology, Los Angeles, California, United States \\ 'Zhejiang University School of Medicine, Sir Run Run Shaw Hospital, Hangzhou, China \\ 'Zhejiang University, Institute of Translational Medicine, Hangzhou, China
}

\begin{abstract}
Dual-energy computed tomography (CT) has the potential to decompose tissues into different materials. However, the classic direct inversion (DI) method for multimaterial decomposition (MMD) cannot accurately separate more than two basis materials due to the ill-posed problem and amplified image noise. We propose an integrated MMD method that addresses the piecewise smoothness and intrinsic sparsity property of the decomposition image. The proposed MMD was formulated as an optimization problem including a quadratic data fidelity term, an isotropic total variation term that encourages image smoothness, and a nonconvex penalty function that promotes decomposition image sparseness. The mass and volume conservation rule was formulated as the probability simplex constraint. An accelerated primal-dual splitting approach with line search was applied to solve the optimization problem. The proposed method with different penalty functions was compared against DI on a digital phantom, a Catphan ${ }^{\circledR} 600$ phantom, a quantitative imaging phantom, and a pelvis patient. The proposed framework distinctly separated the CT image up to 12 basis materials plus air with high decomposition accuracy. The cross talks between two different materials are substantially reduced, as shown by the decreased nondiagonal elements of the normalized cross correlation (NCC) matrix. The mean square error of the measured electron densities was reduced by $72.6 \%$. Across all datasets, the proposed method improved the average volume fraction accuracy from $61.2 \%$ to $99.9 \%$ and increased the diagonality of the NCC matrix from 0.73 to 0.96. Compared with $\mathrm{DI}$, the proposed MMD framework improved decomposition accuracy and material separation. ๑ 2019 Society of Photo-Optical Instrumentation Engineers (SPIE) [DOI: 10.1117/1.JMI.6.4.044004]
\end{abstract}

Keywords: dual-energy computed tomography; multimaterial decomposition; primal-dual splitting; nonconvex optimization.

Paper 19096R received Apr. 8, 2019; accepted for publication Sep. 23, 2019; published online Oct. 15, 2019.

\section{Introduction}

Conventional x-ray computed tomography (CT) projections are acquired with a single-energy spectrum. The reconstructed single-energy CT (SECT) provides the linear attenuation coefficients (LACs) of the scanning object, but the LACs depend on both the effective atomic number and the electron density, making it insufficient to determine the material components. Dualenergy CT (DECT) is acquired with two distinct energy spectra that are attenuated differently by the tissues. Therefore, DECT provides enhanced information to better differentiate and quantify material compositions. DECT shows promise in many clinical applications, including virtual unenhancement imaging, ${ }^{1,2}$ liver lesion characterization, ${ }^{3}$ kidney stone characterization, ${ }^{4}$ oncologic imaging, ${ }^{1,5}$ bone removal, ${ }^{1}$ etc.

Despite the potential of using DECT for multimaterial decomposition (MMD), the problem is ill-defined for decomposing more than two materials without additional assumption. ${ }^{6,7}$ Existing DECT MMD approaches can be classified into three categories: projection-based method, integrated method, and image-based method. The projection-based method decomposes the two independent sinograms into two basis components by interpolating the lookup table and then performs separate image reconstruction. ${ }^{8}$ The integrated method incorporates the DECT acquisition into the forward projection model and then reconstructs basis material images directly from the DECT projections. ${ }^{9}$ The projection- based method and integrated method are robust to beam hardening artifacts, ${ }^{8,9}$ but there are several limitations. First, the projection-based method is limited to decomposing two basis materials. It is neither straightforward nor desirable to introduce additional constraints in the projection domain. Second, the projection-based method requires strict spatial and temporal consistency between the high-energy (HE) and low-energy (LE) acquisition. The condition is not met by many DECT systems using dual-source or single-source with fast kilovoltage-switching. Third, the integrated method is computationally expensive due to the repeated projection and backprojection during the reconstruction of basis image components. Fourth, the coupling between the reconstruction and image decomposition in the integrated method, especially with multiple basis materials, leads to decomposition results that are sensitive to parameter tuning.

Alternatively, image-based decomposition methods were investigated, which perform basis material decomposition on the HE and LE CT images. This method is applicable to all DECT systems, straightforward, and computationally tractable. Mendonça et al. ${ }^{10}$ proposed an image-based MMD method that introduces two assumptions: mass and volume conservation, and that there are no more than three materials in each voxel. For each voxel, the method loops over a material triplet library, identifies the best-fit triplet, and decomposes the voxel into the three materials via direct matrix inversion. The method is termed direct inversion (DI), and it demonstrates the capability of 
decomposing CT into more than three basis materials. However, the decomposition accuracy is sensitive to the selection of material triplet library, which limits the flexibility of MMD, and the resultant images suffer from substantially amplified noise.

In this study, we propose a DECT MMD method that decomposes the CT volume into multiple basis materials accurately while suppressing the decomposition image noise. Instead of rigidly confining each voxel to contain at most three basis materials, a nonconvex sparsity term is introduced to penalize the number of materials that are simultaneously present in the same voxel.

\section{Method}

\subsection{Formulation}

The image domain DECT MMD problem is formulated as follows:

$\underset{x}{\operatorname{minimize}} \frac{1}{2}\|A x-b\|_{2}^{2}+\lambda \operatorname{TV}(x)+\eta \sum_{k, i}\left|x_{k i}\right|^{\alpha}$

subject to $x \in S_{P}$,

where the optimization variable $x$ is the volume fraction (VF) matrix of dimension $N_{k}$ by $N_{i}$. The voxels on the component image are indexed by $i$, and the decomposition materials are indexed by $k$. $x_{k i}$ is the VF of the $k^{\prime}$ th material for the $i$ 'th voxel. $\mathbf{A}$ is the component-to-attenuation transformation matrix defined as

$\mathbf{A}=\left[\begin{array}{llll}\mu_{1 l} & \mu_{2 l} & \cdots & \mu_{N_{k} l} \\ \mu_{1 h} & \mu_{2 h} & \cdots & \mu_{N_{k} h}\end{array}\right]$,

where $\mu_{k l}$ and $\mu_{k h}$ are the LACs of the $k$ 'th material at HE and LE, respectively. Matrix A transforms the VF into the LACs at $\mathrm{HE}$ and LE for each voxel. $b$ is the attenuation image at HE and LE from measurements, defined as

$b=\left[\begin{array}{llll}b_{1 l} & b_{2 l} & \ldots & b_{N_{i} l} \\ b_{1 h} & b_{2 h} & \ldots & b_{N_{i} h}\end{array}\right]$

where $b_{i l}$ and $b_{i h}$ denotes the LAC of the $i$ 'th voxel on the CT image at LE and $\mathrm{HE}$, respectively. $\mathrm{TV}(x)$ is the isotropic total variation regularization, defined as

$\mathrm{TV}(x)=\sum_{i k} \sqrt{\left(\nabla_{r} x\right)_{i k}^{2}+\left(\nabla_{c} x\right)_{i k}^{2}}$

where $\nabla_{r}$ and $\nabla_{c}$ are the derivative matrix along the row and column directions, respectively. $S_{P}$ is the probability simplex set that enforces the mass and volume conservation rule on each voxel, such that each column of the VF matrix $x$ satisfies the sum-to-one and non-negative constraints:

$x \in S_{P} \leftrightarrow\left\{\begin{array}{ll}x_{k i} \geq 0, & \forall i, k \\ \sum_{k} x_{k i}=1, & \forall i\end{array}\right.$.

The first term is the quadratic data fidelity term that minimizes the difference between the measured DECT image and the estimated DECT image calculated from the VF matrix $x$. The second term is the isotropic TV regularization term, applied on each component image to encourage image smoothness while preserving image edges. The last term is the penalty function in the form of $|\cdot|^{\alpha}(0 \leq \alpha \leq 1)$, which promotes sparsity on the number of materials that simultaneous present in the same voxel. In this study, we specifically focus on four cases with $\alpha=0, \frac{1}{2}, \frac{2}{3}, 1$. In the case of $\alpha=1$, the sparsity term reduces to a constant under the mass and volume conservation constraint $\left(x \in S_{P}\right)$. In other cases, the penalty functions are nonconvex with the nonconvexity increases as $\alpha$ goes to 0 .

\subsection{Algorithm}

This study utilizes an accelerated primal-dual splitting approach with line search for both convex and nonconvex problems, ${ }^{11,12}$ which solves the optimization problem of the form:

minimize $F(K x)+G(x)$,

where $G$ is convex, $F$ possibly nonconvex, and $K$ a linear operator.

The optimization problem in Eq. (1) is formulated into the canonical form shown in Eq. (3) by defining

$K=\left[\begin{array}{c}A \\ D \\ I\end{array}\right], \quad G(x)=I_{S_{p}}(x)= \begin{cases}\infty, & x \notin S_{p} \\ 0, & x \in S_{p}\end{cases}$

$F\left(\left[\begin{array}{l}\hat{z}_{1} \\ \hat{z}_{2} \\ \hat{z}_{3}\end{array}\right]\right)=F_{1}\left(\hat{z}_{1}\right)+F_{2}\left(\hat{z}_{2}\right)+F_{3}\left(\hat{z}_{3}\right)$

$F_{1}\left(\hat{z}_{1}\right)=\frac{1}{2}\left\|\hat{z}_{1}-b\right\|_{2}^{2}$,

$F_{2}\left(\hat{z}_{2}\right)=\lambda\left\|\hat{z}_{2}\right\|_{1}$

$\left(F_{3}\left(\hat{z}_{3}\right)\right)_{i}=\eta\left|\hat{z}_{3 i}\right|^{\alpha}$.

$F(K x)$ is equivalent to the objective function in Eq. (1). $G(x)$ is an indicator function that equals to 0 if $x \in S_{p}$ and infinity if $x \notin S_{p}$, which enforces the sum-to-one and non-negative constraints.

The accelerated primal-dual algorithm with line search ${ }^{12}$ is presented in Algorithm 1, where the key steps are the evaluations of the proximal operator of functions $G$ and $F$. The proximal operator of a function $H$ with step size $t$ is defined as follows: ${ }^{13}$

$\operatorname{Prox}_{t H}(z)=\underset{x}{\arg \min }\left(H(x)+\frac{1}{2 t}\|x-z\|_{2}^{2}\right)$.

Following the definition, the proximal operator of $G$ reduces to the projection onto the probability simplex $S_{p}$, which can be efficiently solved by sorting and thresholding the input vector, as presented in Algorithm 2. ${ }^{14}$

With the separable sum rule, the evaluation of the proximal operator of $F$ reduces to evaluating the proximal operator with respect to each variable:

$\operatorname{Prox}_{w F}\left(\left[\begin{array}{l}\hat{z}_{1} \\ \hat{z}_{2} \\ \hat{z}_{3}\end{array}\right]\right)=\left(\left[\begin{array}{l}\operatorname{Prox}_{w F_{1}}\left(\hat{z}_{1}\right) \\ \operatorname{Prox}_{w F_{2}}\left(\hat{z}_{2}\right) \\ \operatorname{Prox}_{w F_{3}}\left(\hat{z}_{3}\right)\end{array}\right]\right)$. 
Algorithm 1 Accelerated primal-dual algorithm with line search.

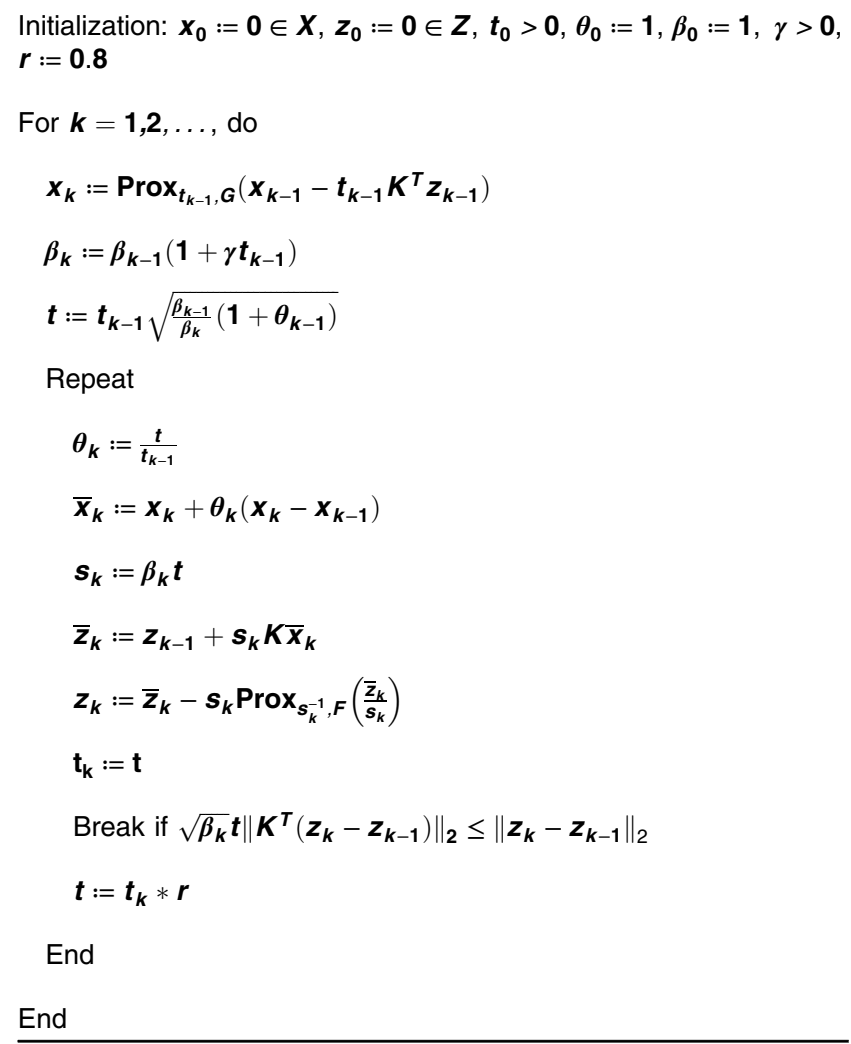

Algorithm 2 Proximal operator evaluation of $\boldsymbol{G}\left(\operatorname{Prox}_{\boldsymbol{t} \boldsymbol{G}}(\boldsymbol{x})\right)$.

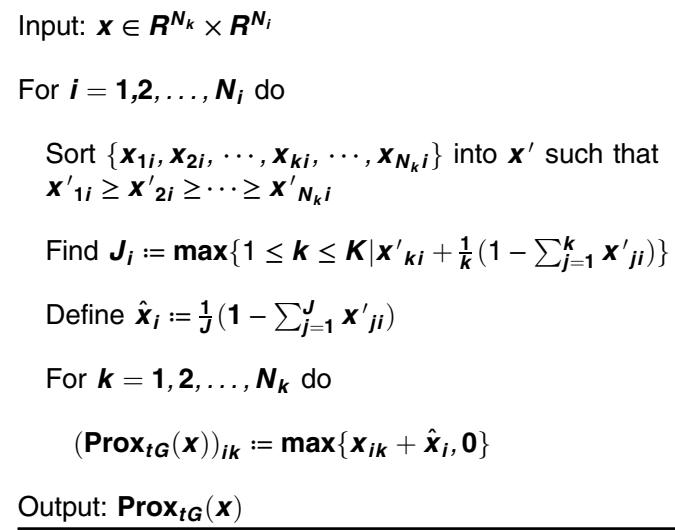

Following the definition of proximal operator in Eq. (4), the proximal operators of $F_{1}$ and $F_{2}$ are

$$
\begin{aligned}
& \operatorname{Prox}_{w F_{1}}\left(\hat{z}_{1}\right)=\frac{\hat{z}_{1}+w b}{w+1}, \\
& \left(\operatorname{Prox}_{w F_{2}}\left(\hat{z}_{2}\right)\right)_{i k}=\left\{\begin{array}{ll}
\left(1-\frac{w \lambda}{\left\|\left(\hat{z}_{2}\right)_{i k}\right\|_{2}}\right)\left(\hat{z}_{2}\right)_{i k}, & \left\|\left(\hat{z}_{2}\right)_{i k}\right\|_{2}>w \lambda \\
0, & \left\|\left(\hat{z}_{2}\right)_{i k}\right\|_{2} \leq w \lambda
\end{array} .\right.
\end{aligned}
$$

Due to the separability, the proximal operator of $F_{3}$ reduces to pointwise proximal operator evaluation of $\eta|\cdot|^{\alpha}$, as follows:
$\left(\operatorname{Prox}_{w F_{3}}\left(\hat{z}_{3}\right)\right)_{i}=\underset{x}{\operatorname{argmin}}\left(\eta|x|^{\alpha}+\frac{1}{2 w}\left(x-\hat{z}_{3 i}\right)^{2}\right)$.

Exact analytic solutions to Eq. (5) exist for the scenarios considered in this study $\left(\alpha=0, \frac{1}{2}, \frac{2}{3}, 1\right)$, which can be found in Sec. 6.1. For other values of $\alpha$, the proximal operators could be evaluated numerically with an iterative approach, such as Newton's method. ${ }^{15}$

With the evaluations of the proximal operator of functions $G$ and $F$, the DECT MMD optimization problem in Eq. (1) could be solved following Algorithm 1.

\subsection{Evaluation}

The proposed framework with different sparsity parameters in the penalty functions was evaluated on a digital phantom, a Catphan ${ }^{\circledR} 600$ phantom, a quantitative imaging phantom, and a pelvis patient, and is compared with the DI method. ${ }^{10}$ The quantitative imaging phantom data were acquired on a Siemens SOMATOM Force DECT. The Catphan and pelvis patient data were acquired on a Siemens SOMATOM Definition Flash. For the pelvis patient data, the mAs and CT dose index for the [LE, $\mathrm{HE}$ ] acquisitions were [170 $\mathrm{mAs}, 131 \mathrm{mAs}$ ] and [4.92 $\mathrm{mGy}$, 3.66 mGy], respectively. The LE and HE CT images, as shown in Figs. 1, 3, 5, and 7, were reconstructed using the standard filtered backprojection (FBP). The HE LAC and LE LAC of the basis material were computed as the average values of the $\mathrm{HE}$ LACs and LE LACs of the region of interest (ROIs), as shown on the LE CT images for all cases. These LAC values are presented in Table 4 in Sec. 6.

For quantitative evaluation of the material decomposition accuracy, the mean and standard deviation (STD), electron density, as well as the VF, were computed within each ROI on the decomposition component image. The electron density $\rho_{i}$ at voxel $i$ is calculated by

$\rho_{i}=\sum_{k=1}^{N_{k}} x_{k i} \rho(k)$

where $\rho(k)$ is the electron density of the $k^{\prime}$ th material. The VF accuracy in a uniform ROI is defined as follows:

$\mathrm{VF}=\left(1-\frac{\left\|\bar{x}-x_{g}\right\|_{2}}{\left\|x_{g}\right\|_{2}}\right) \times 100 \%$

where $\bar{x}$ is the mean material component vector over all voxels within the uniform ROI and $x_{g}$ is the material component vector of the ground truth decomposition.

To quantify the amount of overlap between different material decompositions across the whole image, the normalized cross correlation (NCC) coefficients at zero lag are evaluated for every pair of materials. The NCC coefficient at zero lag of material $k_{1}$ and $k_{2}$ is defined as follows:

$$
R_{k_{1} k_{2}}=\frac{\sum_{i=1}^{N_{i}} x_{k_{1} i} x_{k_{2} i}}{\sqrt{\sum_{i=1}^{N_{i}} x_{k_{1} i}^{2}} \sqrt{\sum_{i=1}^{N_{i}} x_{k_{2} i}^{2}}}
$$

which equals to 0 if the two materials are completely separated and equals to 1 if they have identical distribution on the whole image. The NCC matrix is defined as a matrix with entries of 
$R_{k_{1} k_{2}}$. The diagonal elements of the NCC matrix are equal to 1 by definition, and the other elements are between 0 and 1 , indicating the extent of material separation. If all materials are completely separated for all voxels, then all the off-diagonal elements should be 0 . The diagonality $D$ of the NCC matrix is computed using the Pearson correlation coefficient:

$D=\frac{\left(\sum_{k_{1}, k_{2}} R_{k_{1} k_{2}}\right)\left(\sum_{k_{1}, k_{2}} k_{1} k_{2} R_{k_{1} k_{2}}\right)-\left(\sum_{k_{1}, k_{2}} k_{1} R_{k_{1} k_{2}}\right)\left(\sum_{k_{1}, k_{2}} k_{2} R_{k_{1} k_{2}}\right)}{\sqrt{\left(\sum_{k_{1}, k_{2}} R_{k_{1} k_{2}}\right)\left(\sum_{k_{1}, k_{2}} k_{1}^{2} R_{k_{1} k_{2}}\right)-\left(\sum_{k_{1}, k_{2}} k_{1} R_{k_{1} k_{2}}\right)^{2}} \sqrt{\left(\sum_{k_{1}, k_{2}} R_{k_{1} k_{2}}\right)\left(\sum_{k_{1}, k_{2}} k_{2}^{2} R_{k_{1} k_{2}}\right)-\left(\sum_{k_{1}, k_{2}} k_{2} R_{k_{1} k_{2}}\right)^{2}}}$

where $D$ equals to 1 for the diagonal matrix, -1 for the antidiagonal matrix, and 0 for the uniform matrix. In the case of every voxel on the $\mathrm{CT}$ image being composed of only a single material, the diagonality of the NCC matrix is 1 . On the other hand, in the case where every voxel contains an equal amount of all basis materials, the diagonality of the NCC matrix is 0 . The diagonality $D$ of the NCC matrix summarizes the decomposition separability over the whole image and across all materials.

\section{Results}

\subsection{Digital Phantom}

Figure 1 shows the LE and HE CT image of the digital phantom. This simple digital phantom is made up of four basis materials, including bone, iodine, water, and air, corresponding to the four ROIs indicated by the rectangular area. Figure 2 shows the decomposition results on the digital phantom using the proposed framework with different $\alpha$ values and the classic DI method. In the cases when $\alpha=0, \frac{1}{2}, \frac{2}{3}$, the proposed framework distinctly separated all basis materials and with low noise, achieving a clear border between different materials. On the contrary, neither DI nor the proposed framework with $\alpha=1$ achieved the desired sparsity or assigned the correct material to each ROI. The material separation capability of the proposed framework with $\alpha=0, \frac{1}{2}, \frac{2}{3}$ is further confirmed with the NCC map in Fig. 2, showing little or no cross talks between two materials. With DI, the iodine component is mixed up with the bone and water. In the case of $\alpha=1$, none of the component is separated.

\subsection{Catphan $^{\circledR} 600$ Phantom}

Figure 3 shows the LE and HE CT images of the Catphan ${ }^{\circledR} 600$ phantom with contrast rods, which are made of six basis materials, including Teflon, Delrin, iodine solution of $10 \mathrm{mg} / \mathrm{ml}$, polymethyl pentene (PMP), inner soft tissue, and air, corresponding to the six ROIs on the LE CT image [Fig. 3(a)]. The materials in the labeled rods on the HE CT [Fig. 3(b)] are Teflon, Delrin, iodine solution of $10 \mathrm{mg} / \mathrm{ml}$, polystyrene, lowdensity polyethylene, PMP, and iodine solution of $5 \mathrm{mg} / \mathrm{ml}$, respectively. The VF accuracy was evaluated on the six ROIs, and the electron density was evaluated on the seven contrast rods. Figure 4 shows the decomposition image and the NCC map. The proposed framework with $\alpha=0, \frac{1}{2}, \frac{2}{3}$ successfully separated the phantom into the six basis components with minimal cross talk between different materials. With the classical DI method, the off-diagonal elements of the NCC matrix are up to 0.36 , showing that the two corresponding basis materials, Delrin and PMP, are not well-separated. The proposed method with $\alpha=1$ is unable to achieve material separation at all. Table 1 shows the evaluated electron densities for the seven contrast rods. The mean square error of the electron density was reduced by $72.6 \%$ for the proposed framework with $\alpha=0,1 / 2,2 / 3$ compared with DI.

\subsection{Quantitative Imaging Phantom}

Figure 5 show the DECT image for the quantitative imaging phantom, which consists of 12 basis materials, including $2 \mathrm{mg} / \mathrm{ml}$ iodine solution (ROI1), $5 \mathrm{mg} / \mathrm{ml}$ iodine solution (ROI2), $10 \mathrm{mg} / \mathrm{ml}$ iodine solution (ROI3), $15 \mathrm{mg} / \mathrm{ml}$ iodine

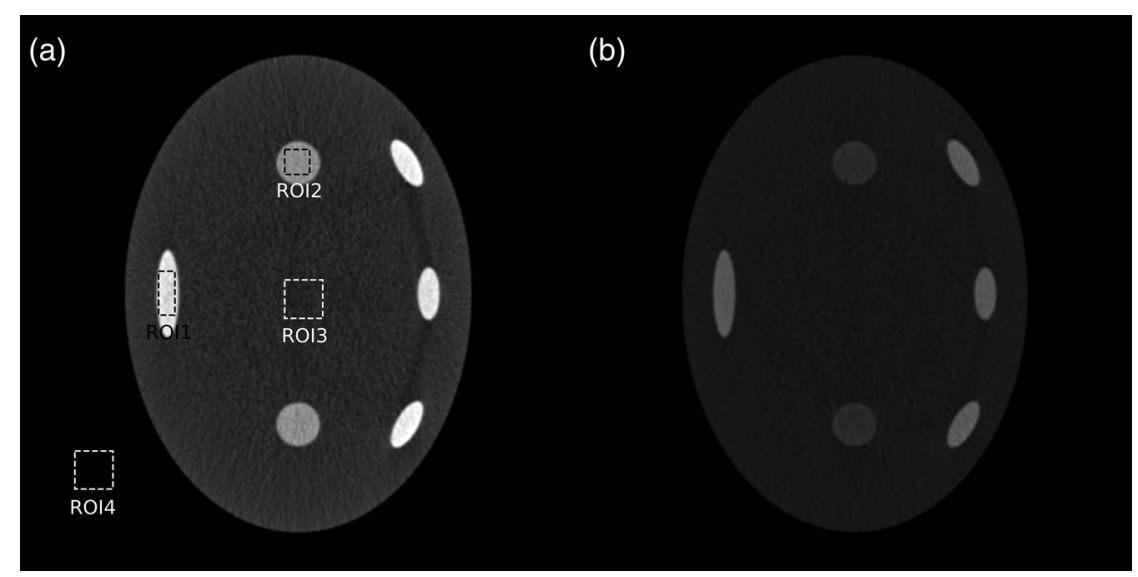

Fig. 1 (a) The LE: $75 \mathrm{kVp}$ and (b) the HE: $140 \mathrm{kVp}$ CT image of the digital phantom. The components of the ROls are bone (ROI1), iodine (ROI2), water (ROI3), and air (ROI4). The displaying window is $[0.01,0.065] \mathrm{mm}^{-1}$. 


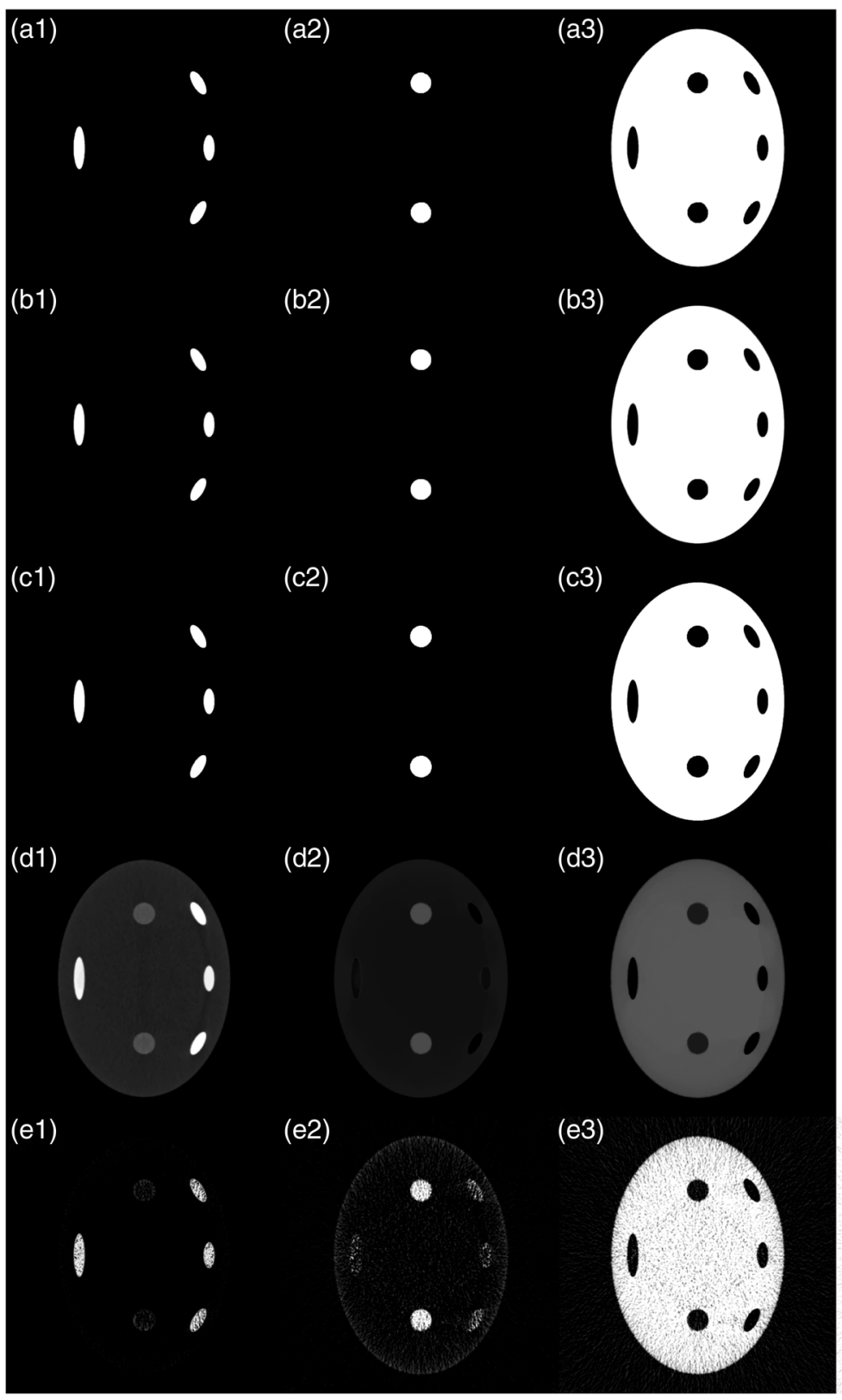

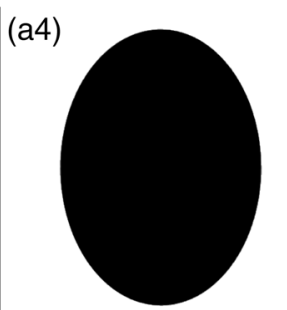

(b4)
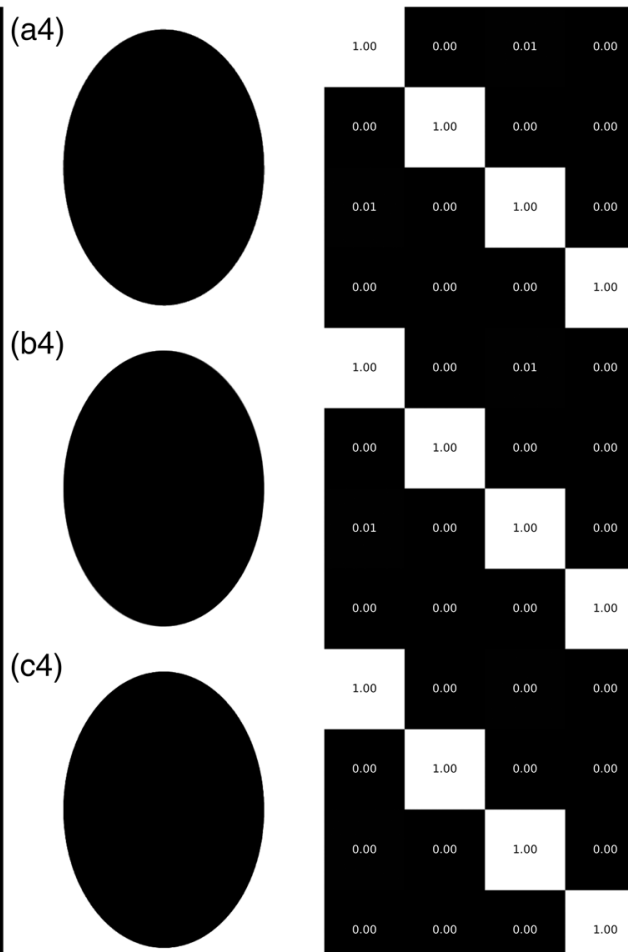

(d4)

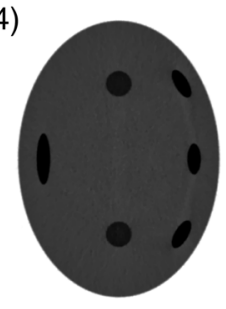

(e4)

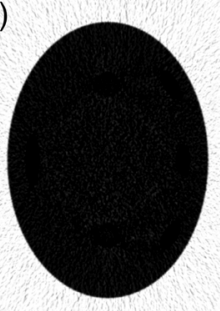

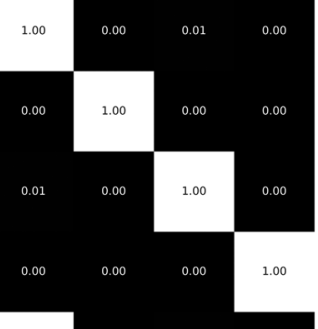
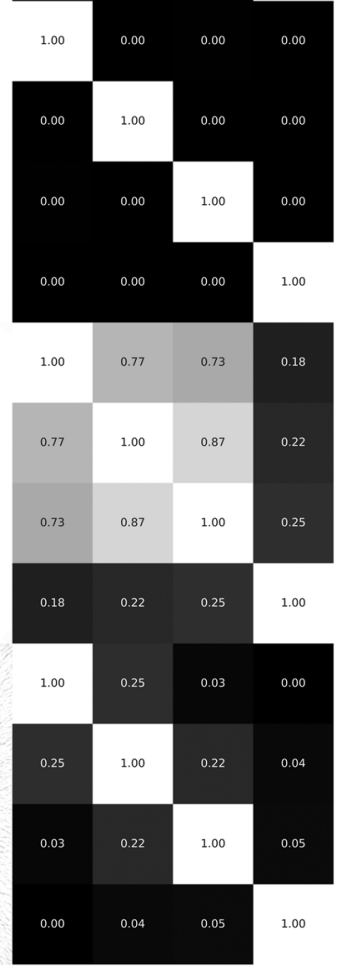

Fig. 2 Decomposition component images of (1) bone, (2) iodine, (3) water, and (4) air, decomposed using the proposed framework when (a) $\alpha=0$, (b) $\alpha=\frac{1}{2}$, (c) $\alpha=\frac{2}{3}$, (d) $\alpha=1$, and (e) the DI method. The last column is the $4 \times 4 \mathrm{NCC}$ map of the decomposition in the same row, with each square showing the corresponding entries in the NCC matrix, where the basis materials are bone, iodine, water, and air, from top to bottom and from left to right.

solution (PMP) (ROI4), $50 \mathrm{mg} / \mathrm{ml}$ calcium solution (ROI5), $100 \mathrm{mg} / \mathrm{ml}$ calcium solution (ROI6), $300 \mathrm{mg} / \mathrm{ml}$ calcium solution (ROI7), HE blood 70 (ROI8), HE blood 100 (ROI9), adipose (ROI10), water (ROI1), and brain (ROI12). In this phantom study, to avoid being trapped in undesired local minima with the increased number of basis materials, the algorithm is initialized by setting VF $=1$ for the basis material that is closest to each pixel. Figure 6 shows the decomposition images with the corresponding NCC map. The proposed framework with $\alpha=0, \frac{1}{2}, \frac{2}{3}$ decomposed the DECT images into 12 different basis components plus air and improved the VF accuracy from $51 \%$ using DI method to $100 \%$. The nondiagonal entries of the NCC map are close to 0 for the proposed framework with $\alpha=0, \frac{1}{2}, \frac{2}{3}$, showing clean separation of the basis materials. The off- diagonal entries are close to 1 for the $\alpha=1$ scenario. The classical DI method is unable to separate similar materials. For example, the HE blood 100 (ROI9), adipose (ROI10), and water (ROI11) have similar LAC values, resulting in substantial nonzero NCC elements $R_{k_{1} k_{2}}$ using DI.

\subsection{Pelvis Patient}

Figures 7 and 8 show the DECT image and the decomposition images with the corresponding NCC map, respectively, for the pelvis patient. The proposed framework with $\alpha=0, \frac{1}{2}, \frac{2}{3}$ decomposed the DECT images into bone (ROI1), iodine (ROI2), muscle (ROI3), fat (ROI4), and air, and achieved an NCC matrix with nondiagonal coefficients close to 0 . 


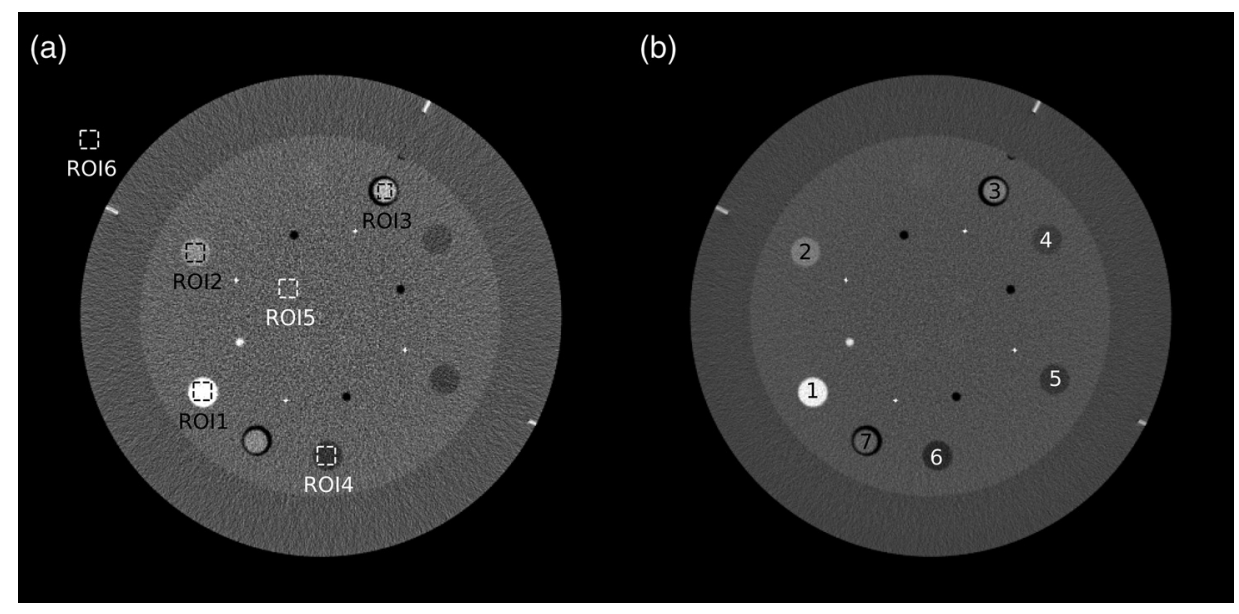

Fig. 3 (a) The LE: $75 \mathrm{kVp}$ and (b) the HE: $125 \mathrm{kVp}$ CT image of the Catphan ${ }^{\circledR} 600$ phantom. The material components of the ROls on the low energy CT image are Teflon (ROI1), Delrin (ROI2), iodine of $10 \mathrm{mg} / \mathrm{ml}$ (ROI3), PMP (ROI4), inner soft tissue (ROI5), and air (ROI6). The labeled contrast rods on the HE CT image are composed of (1) Teflon, (2) Delrin, (3) iodine solution of $10 \mathrm{mg} / \mathrm{ml}$, (4) polystyrene, (5) low-density polyethylene, (6) PMP, and (7) iodine solution of $5 \mathrm{mg} / \mathrm{ml}$. The displaying window is $[0.01,0.04] \mathrm{mm}^{-1}$.

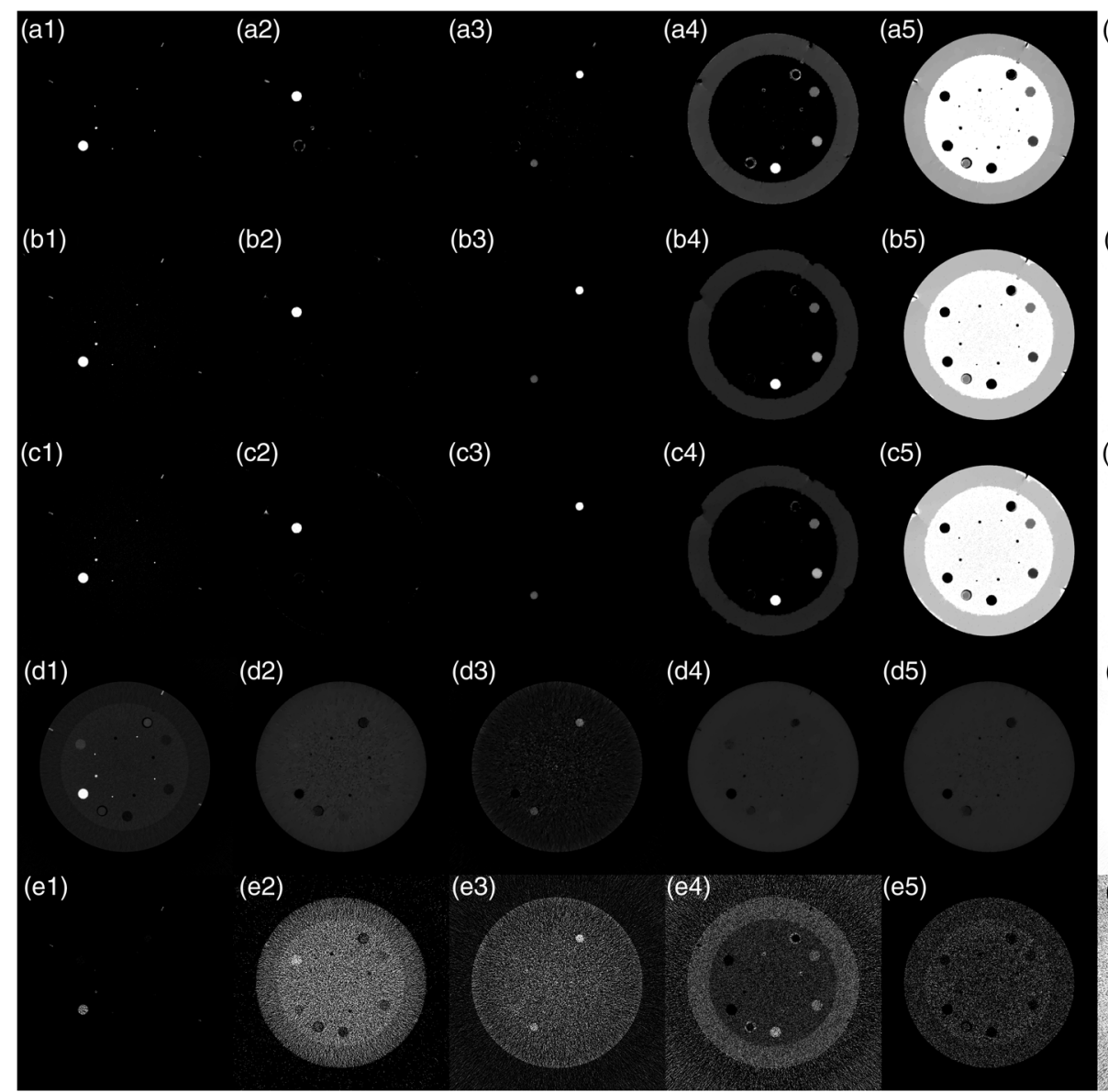

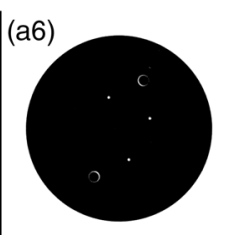

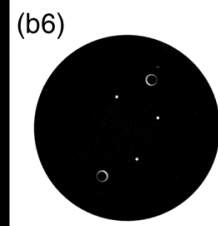

(c6)

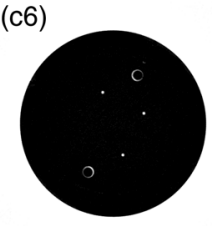

(d6)
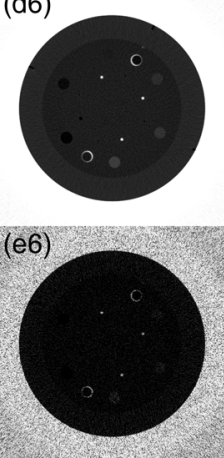
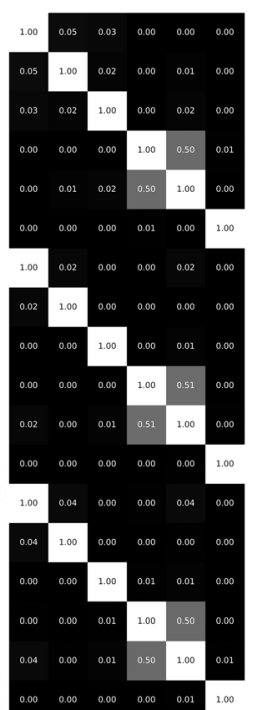

\begin{tabular}{lll|lll}
1.00 & 0.91 & 0.74 & 0.90 & 0.90 \\
\hline
\end{tabular}

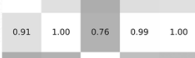

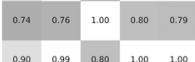

$\begin{array}{lllll}0.90 & 0.99 & 0.90 & 1.00 & 1.00 \\ 0.90 & 1.00 & 0.79 & 1.00 & 1.00\end{array}$
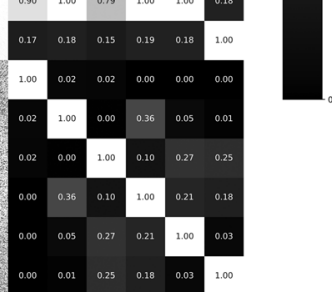

Fig. 4 Decomposition component images of (1) Teflon, (2) Delrin, (3) iodine solution of $10 \mathrm{mg} / \mathrm{ml}$, (4) PMP, (5) inner soft tissue (LDPE), (6) air, decomposed using the proposed framework when (a) $\alpha=0$, (b) $\alpha=\frac{1}{2}$, (c) $\alpha=\frac{2}{3}$, and (d) $\alpha=1$; (e) the DI method. The last column is the $6 \times 6$ NCC map of the decomposition in the same row, with each square showing the corresponding entries in the NCC matrix, where the basis materials are Teflon, Delrin, iodine solution of $10 \mathrm{mg} / \mathrm{ml}, \mathrm{PMP}, \mathrm{LDPE}$, and air, from top to bottom and from left to right. 
Table 1 The electron densities measured on the Catphan contrast rods labeled in Fig. 3(b). The RMSE is evaluated for each method as the mean square error of the seven rods.

\begin{tabular}{|c|c|c|c|c|c|c|c|c|c|}
\hline Method & & $\begin{array}{l}\text { Rod } 1 \\
\text { Teflon }\end{array}$ & $\begin{array}{l}\text { Rod } 2 \\
\text { Delrin }\end{array}$ & $\begin{array}{c}\text { Rod } 3 \\
\text { lodine } \\
(10 \mathrm{mg} / \mathrm{ml})\end{array}$ & $\begin{array}{c}\text { Rod } 4 \\
\text { polystyrene }\end{array}$ & $\begin{array}{l}\text { Rod } 5 \\
\text { LDPE }\end{array}$ & $\begin{array}{l}\text { Rod } 6 \\
\text { PMP }\end{array}$ & $\begin{array}{c}\text { Rod } 7 \\
\text { lodine } \\
(5 \mathrm{mg} / \mathrm{ml})\end{array}$ & $\begin{array}{c}\text { Average } \\
\text { NMSE }\end{array}$ \\
\hline Ground truth & $\rho_{\mathrm{e}}$ & 6.240 & 4.525 & 3.368 & 3.400 & 3.155 & 2.851 & 3.356 & 1 \\
\hline \multirow[t]{2}{*}{$\alpha=0$} & $\rho_{\mathrm{e}}$ & 6.240 & 4.525 & 3.368 & 3.104 & 3.008 & 2.851 & 3.354 & $1.92 \%$ \\
\hline & NMSE & $0.00 \%$ & $0.00 \%$ & $0.00 \%$ & $8.70 \%$ & $4.67 \%$ & $0.00 \%$ & $0.06 \%$ & \\
\hline \multirow[t]{2}{*}{$\alpha=1 / 2$} & $\rho_{\mathrm{e}}$ & 6.218 & 4.514 & 3.357 & 3.119 & 2.997 & 2.854 & 3.352 & $2.06 \%$ \\
\hline & NMSE & $0.35 \%$ & $0.24 \%$ & $0.33 \%$ & $8.26 \%$ & $5.01 \%$ & $0.12 \%$ & $0.13 \%$ & \\
\hline \multirow[t]{2}{*}{$\alpha=2 / 3$} & $\rho_{\mathrm{e}}$ & 6.211 & 4.507 & 3.346 & 3.113 & 2.984 & 2.856 & 3.346 & $2.27 \%$ \\
\hline & NMSE & $0.46 \%$ & $0.41 \%$ & $0.66 \%$ & $8.43 \%$ & $5.43 \%$ & $0.16 \%$ & $0.30 \%$ & \\
\hline \multirow[t]{2}{*}{$\alpha=1$} & $\rho_{\mathrm{e}}$ & 6.169 & 4.203 & 4.011 & 2.961 & 2.770 & 2.509 & 3.578 & $10.16 \%$ \\
\hline & NMSE & $1.14 \%$ & $7.11 \%$ & $19.11 \%$ & $12.91 \%$ & $12.22 \%$ & $12.01 \%$ & $6.63 \%$ & \\
\hline \multirow[t]{2}{*}{ DI } & $\rho_{\mathrm{e}}$ & 4.847 & 4.174 & 3.699 & 3.251 & 3.134 & 2.887 & 3.523 & $7.32 \%$ \\
\hline & NMSE & $22.33 \%$ & $7.76 \%$ & $9.83 \%$ & $4.38 \%$ & $0.67 \%$ & $1.28 \%$ & $4.96 \%$ & \\
\hline
\end{tabular}

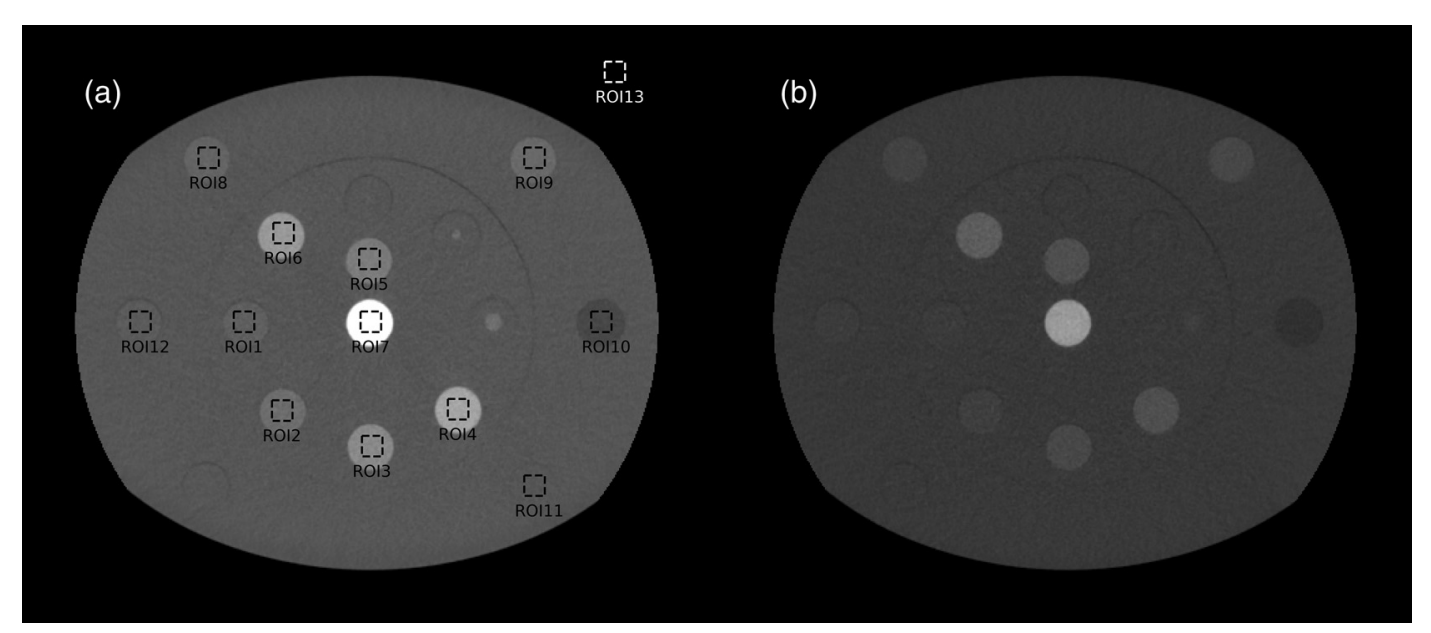

Fig. 5 (a) The LE: $100 \mathrm{kVp}$ and (b) the HE: $150 \mathrm{kVp}$ CT image of the quantitative imaging phantom. The material components of the ROls are $2 \mathrm{mg} / \mathrm{ml}$ iodine solution (ROl1), $5 \mathrm{mg} / \mathrm{ml}$ iodine solution (ROI2), $10 \mathrm{mg} / \mathrm{ml}$ iodine solution (ROI3), $15 \mathrm{mg} / \mathrm{ml}$ iodine solution (PMP) (ROI4), $50 \mathrm{mg} / \mathrm{ml}$ calcium solution (ROI5), $100 \mathrm{mg} / \mathrm{ml}$ calcium solution (ROI6), $300 \mathrm{mg} / \mathrm{ml}$ calcium solution (ROI7), HE blood 70 (ROI8), HE blood 100 (ROI9), adipose (ROI10), water (ROI11), brain (ROI12), and air (ROI13).

Table 2 summarizes the VF accuracy and diagonality of the NCC matrix for all datasets. The proposed method with $\alpha=0, \frac{1}{2}, \frac{2}{3}$ achieves a significantly higher VF accuracy and diagonality than the comparison methods and approaching nearly perfect material decomposition. Across all datasets, the proposed method improved the average VF accuracy from $61.2 \%$ to $99.9 \%$ and increased the diagonality of the NCC matrix from 0.73 to 0.96 .

Table 3 presents the runtime and the hyperparameters used in all cases. Despite that our algorithm used MATLAB built-in GPU computing tools for acceleration, the proposed framework takes 12 min on average, which is slower than the DI method, requiring only 3 min on CPU. The hyperparameters were tuned case-by-case to achieve visually desired sparseness and smoothness. The sparseness and the smoothness can be promoted by increasing $\eta$ and $\lambda$, respectively. $\gamma$ and $t$ are related to the step sizes in the algorithms, which were tuned in a trial-and-error way for faster and more stable convergence.

Figure 9 shows the convergence plots for different $\alpha$ values on the Catphan and the pelvis patient case. The objective values for different cases were not comparable since different hyperparameter values were used in different cases. However, it is worth noting that the plots show different converging patterns. For the convex case $\alpha=1$, the objective goes down nicely with a convex-shaped convergence curve, showing stable and robust convergence. For the nonconvex case $\alpha=0$, the curve goes 


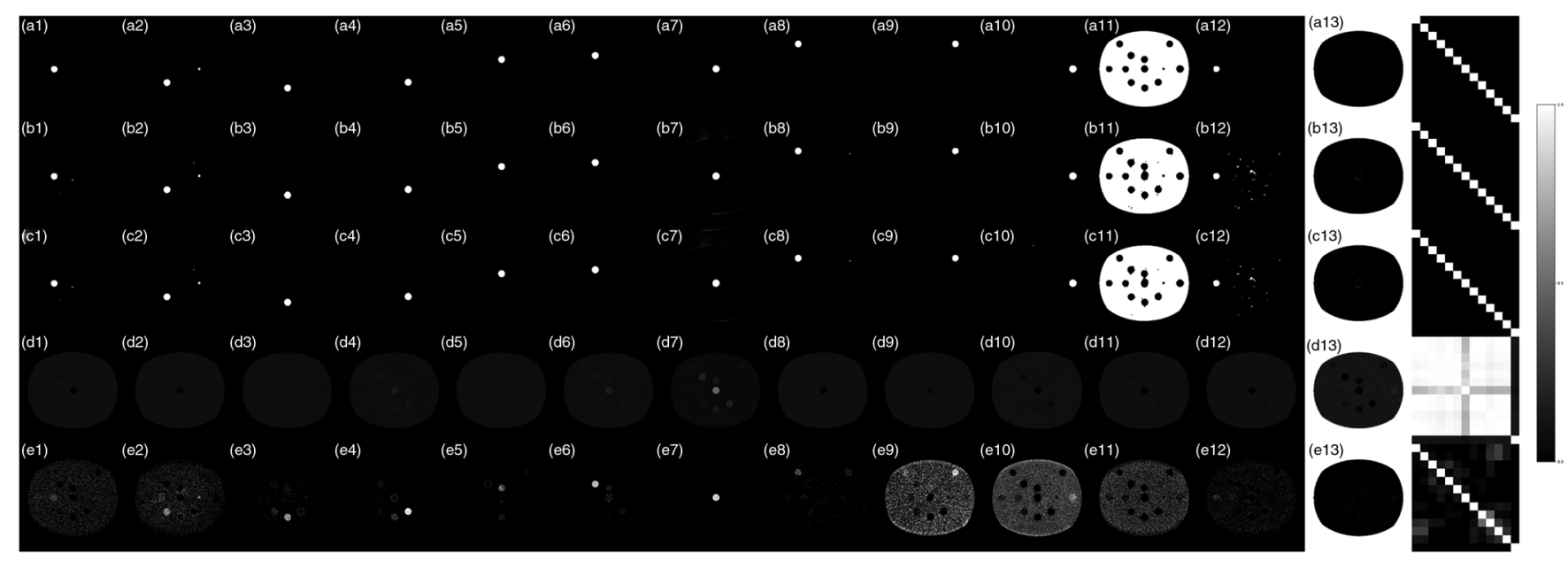

Fig. 6 Decomposition component images of (1) $2 \mathrm{mg} / \mathrm{ml}$ iodine solution, (2) $5 \mathrm{mg} / \mathrm{ml}$ iodine solution (ROI2), (3) $10 \mathrm{mg} / \mathrm{ml}$ iodine solution (ROI3), (4) $15 \mathrm{mg} / \mathrm{ml}$ iodine solution (PMP) (ROI4), (5) $50 \mathrm{mg} / \mathrm{ml}$ calcium solution (ROI5), (6) $100 \mathrm{mg} / \mathrm{ml}$ calcium solution (ROI6), (7) $300 \mathrm{mg} / \mathrm{ml} \mathrm{calcium}$ solution (ROI7), (8) HE blood 70 (ROI8), (9) HE blood 100 (ROI9), (10) adipose (ROI10), (11) water (ROI11), (12) brain (ROI12), and (13) air (ROI13), decomposed using the proposed framework when (a) $\alpha=0$, (b) $\alpha=\frac{1}{2}$, (c) $\alpha=\frac{2}{3}$, and (d) $\alpha=1$, and (e) the DI method. The last column is the NCC map of the corresponding decomposition, with each square showing one NCC matrix element.

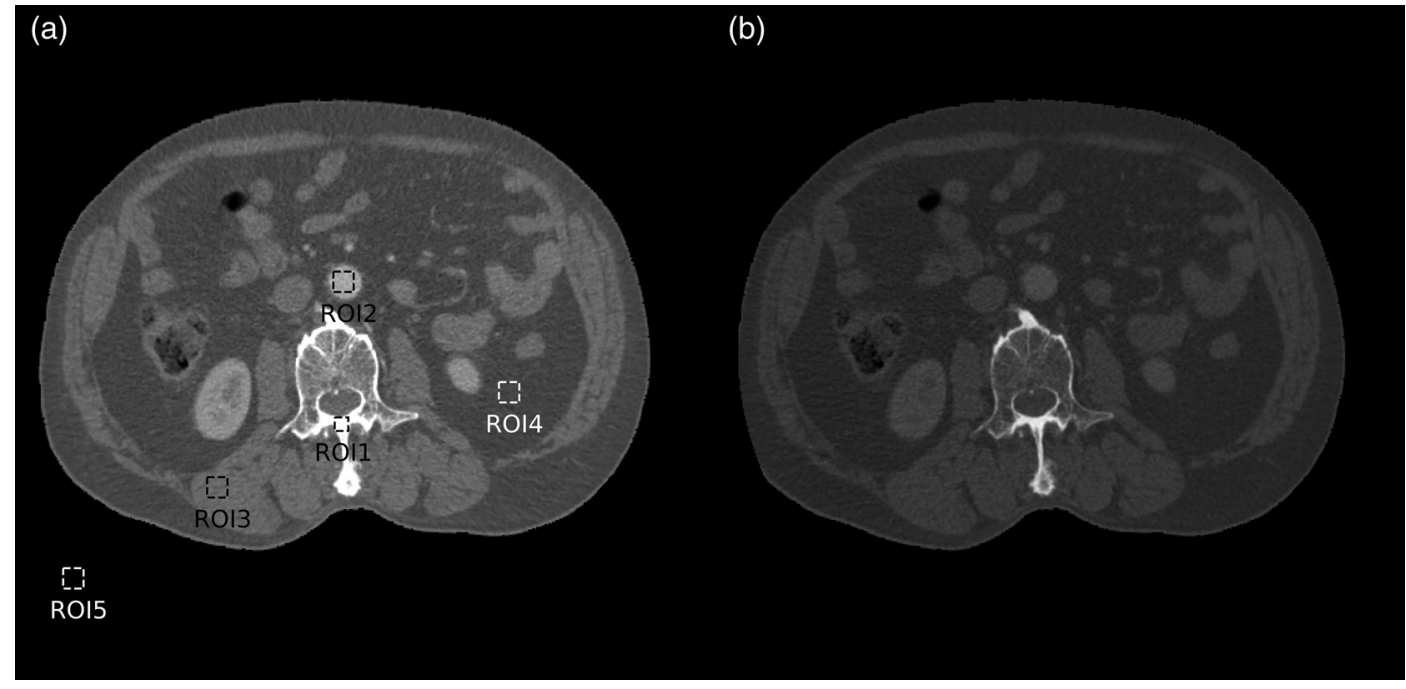

Fig. 7 (a) The LE: $100 \mathrm{kVp}$ and (b) the HE: $140 \mathrm{kVp}$ CT image of the pelvis patient. The material components of the ROls are bone (ROI1), iodine (ROI2), muscle (ROI3), fat (ROI4), and air (ROI5). The displaying window is $[0.01,0.04] \mathrm{mm}^{-1}$.

down with sudden changes. For the $\alpha$ values in between, the $\alpha=\frac{2}{3}$ curve patterns are more similar to that of the $\alpha=1$ curves, whereas the $\alpha=\frac{1}{2}$ curves are more irregular and bumpy.

\section{Discussions}

The standard DI method for DECT-based MMD imposes sparsity constraint by enforcing a hard ceiling of three on the number of materials in each voxel and then solves the basis material components by direct matrix inversion. However, the three-material constraint is arbitrary and unrealistically rigid. Moreover, the direct matrix inversion inevitably amplifies the image noise, as shown in previous publications ${ }^{10}$ and the current study. Our proposed DECT MMD framework utilizes a TV regularization that regulates the decomposition image noise and uses a sparsity regularization to penalize the number of materials that are simultaneously present at the same voxel. The soft sparsity regularization allows the number of basis materials to vary across different voxels.

The sparsity term is in the form of $|x|^{\alpha}(0 \leq \alpha \leq 1)$, where $\alpha=0, \frac{1}{2}, \frac{2}{3}, 1$ were specifically studied. The sparsity term reduces to the $\mathrm{L} 1$ norm of the $x$ with $\alpha=1$ and further reduces to a constant under the mass and volume constraint. Therefore, the sparsity term with $\alpha=1$ does not promote material sparsity despite its desirable mathematical properties of being convex. When $\alpha=0, \frac{1}{2}, \frac{2}{3}$, the corresponding sparsity term has a closedform proximal operator, which could be difficult to evaluate for other values of $\alpha$. The differences between $\alpha=0, \alpha=\frac{1}{2}$, and $\alpha=\frac{2}{3}$ are subtle with respect to the decomposition results. The material penalty term with $\alpha=0$ is also referred to as the counting norm and a mathematically rigorous description of the 


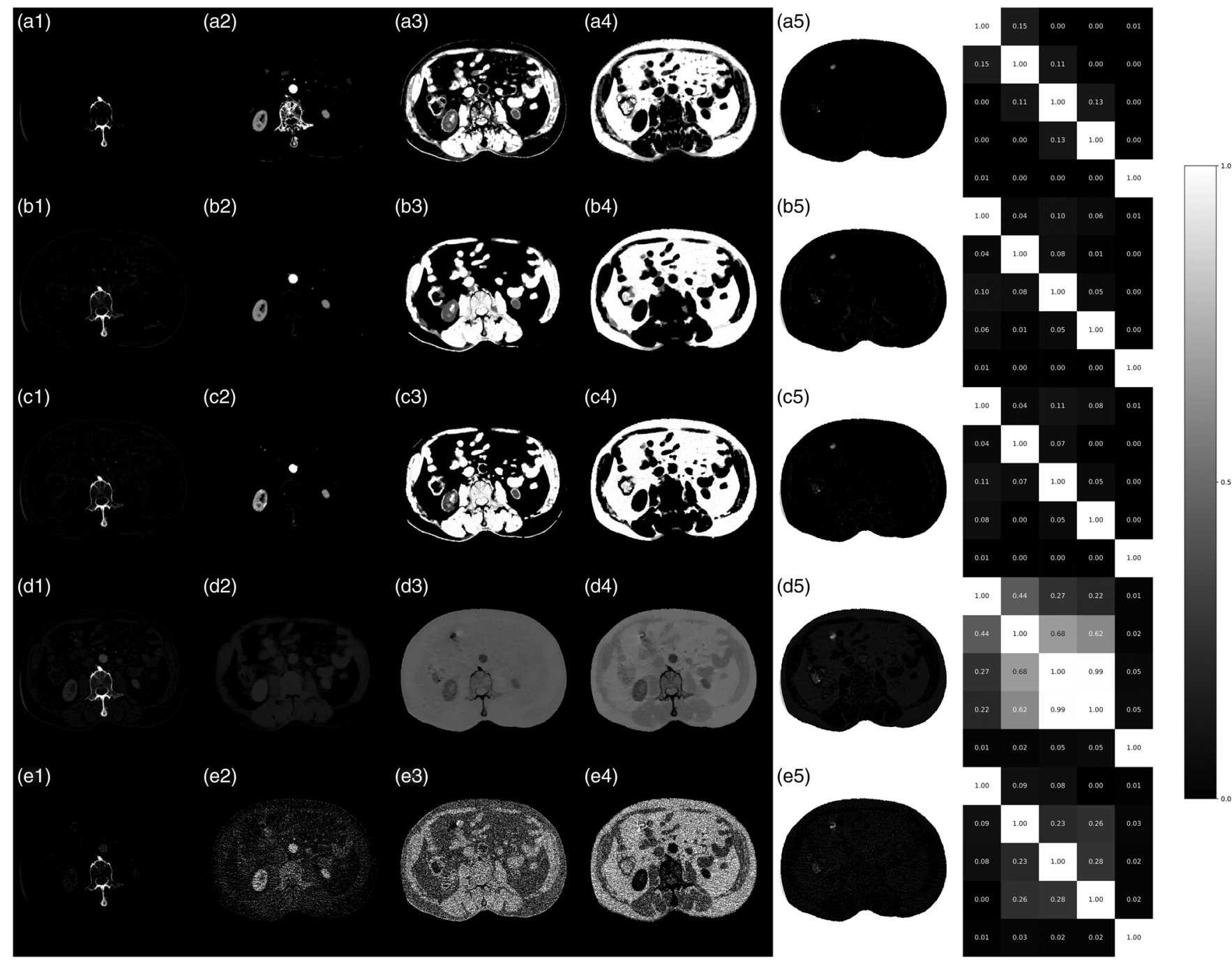

Fig. 8 Decomposition component images of (1) bone (2) iodine (3) muscle (4) fat and (5) air, decomposed using the proposed framework when (a) $\alpha=0$, (b) $\alpha=\frac{1}{2}$, (c) $\alpha=\frac{2}{3}$, and (d) $\alpha=1$; (e) the DI method. The last column is the $5 \times 5$ NCC map of the decomposition in the same row, with each square showing the corresponding entries in the NCC matrix, where the basis materials are bone, iodine, muscle, fat, and air, from top to bottom and from left to right.

Table 2 The VF accuracy and diagonality of the NCC matrix for all datasets.

\begin{tabular}{|c|c|c|c|c|c|}
\hline & & Digital phantom & Catphan & Quantitative imaging phantom & Pelvis patient \\
\hline \multirow[t]{5}{*}{ VF accuracy } & $\alpha=0$ & $100.0 \%$ & $99.9 \%$ & $100 \%$ & NA \\
\hline & $\alpha=1 / 2$ & $100.0 \%$ & $99.6 \%$ & $100 \%$ & \\
\hline & $\alpha=2 / 3$ & $100.0 \%$ & $99.3 \%$ & $100 \%$ & \\
\hline & $\alpha=1$ & $62.1 \%$ & $45.3 \%$ & $15 \%$ & \\
\hline & $\mathrm{DI}$ & $88.4 \%$ & $44.1 \%$ & $51 \%$ & \\
\hline \multirow[t]{5}{*}{ Diagonality } & $\alpha=0$ & 1.00 & 0.95 & 1.00 & 0.95 \\
\hline & $\alpha=1 / 2$ & 0.99 & 0.94 & 0.99 & 0.89 \\
\hline & $\alpha=2 / 3$ & 0.99 & 0.93 & 0.99 & 0.87 \\
\hline & $\alpha=1$ & 0.33 & 0.14 & 0.03 & 0.53 \\
\hline & DI & 0.87 & 0.72 & 0.54 & 0.8 \\
\hline
\end{tabular}


Lyu et al.: Image-domain multimaterial decomposition for dual-energy computed tomography...

Table 3 The runtime and the hyperparameters used in all cases.

\begin{tabular}{|c|c|c|c|c|c|}
\hline & & Digital phantom & Catphan & Quantitative imaging phantom & Pelvis patient \\
\hline \multirow[t]{4}{*}{$\eta$} & $\alpha=0$ & 0.03 & 0.006 & 0.0009 & 0.003 \\
\hline & $\alpha=1 / 2$ & 0.05 & 0.002 & 0.0009 & 0.001 \\
\hline & $\alpha=2 / 3$ & 0.1 & 0.003 & 0.0009 & 0.002 \\
\hline & $\alpha=1$ & 0.5 & 0 & 0.1 & 0 \\
\hline \multirow[t]{4}{*}{$\lambda$} & $\alpha=0$ & 0.05 & 0.002 & 0.02 & 0.0007 \\
\hline & $\alpha=1 / 2$ & 0.25 & 0.003 & 0.005 & 0.0012 \\
\hline & $\alpha=2 / 3$ & 0.05 & 0.0025 & 0.003 & 0.0005 \\
\hline & $\alpha=1$ & 0.01 & 0.0005 & 0 & 0.0005 \\
\hline \multirow[t]{4}{*}{$\gamma$} & $\alpha=0$ & 0.01 & 0.0001 & $1.00 \mathrm{E}-05$ & 0.0001 \\
\hline & $\alpha=1 / 2$ & 0.01 & 0.0001 & $1.00 \mathrm{E}-05$ & 0.0001 \\
\hline & $\alpha=2 / 3$ & 0.001 & 0.001 & 0.001 & $1.00 \mathrm{E}-05$ \\
\hline & $\alpha=1$ & 0.1 & 0.001 & 1 & 0.001 \\
\hline \multirow[t]{4}{*}{$t$} & $\alpha=0$ & 100 & 1 & 1 & 1 \\
\hline & $\alpha=1 / 2$ & 100 & 1 & 1 & 1 \\
\hline & $\alpha=2 / 3$ & 100 & 100 & 0.5 & 100 \\
\hline & $\alpha=1$ & 100 & 100 & 0.01 & 100 \\
\hline \multirow[t]{5}{*}{ Runtime (s) } & $\alpha=0$ & 63.4 & 791.5 & 861.7 & 738.8 \\
\hline & $\alpha=1 / 2$ & 71.2 & 881.4 & 489.1 & 1640.1 \\
\hline & $\alpha=2 / 3$ & 81.5 & 1059.4 & 3542.8 & 962.1 \\
\hline & $\alpha=1$ & 81.3 & 95.1 & 323.9 & 294.7 \\
\hline & DI & 36.2 & 133.6 & 490.1 & 30.9 \\
\hline
\end{tabular}
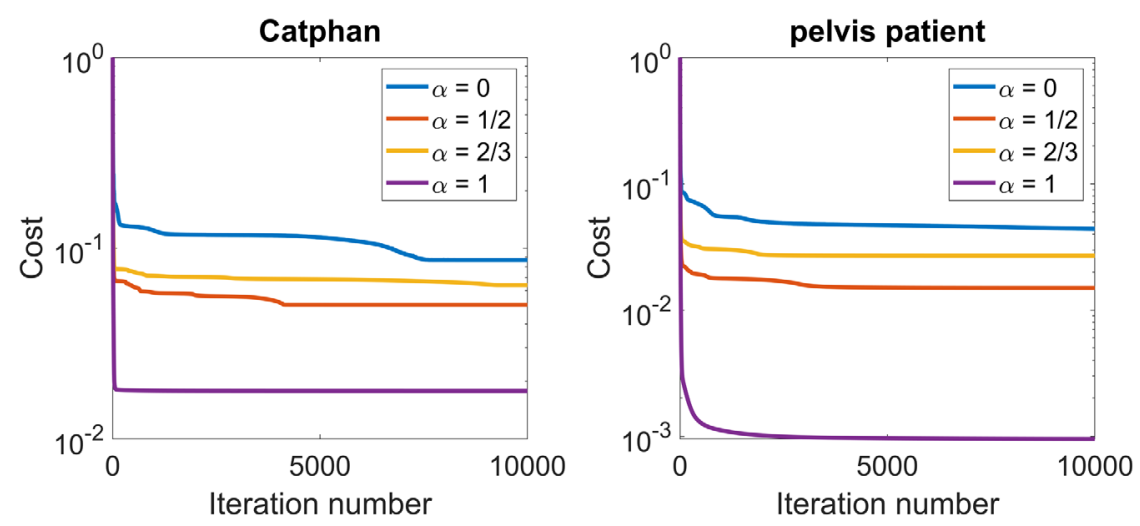

Fig. 9 Convergence plots for different $\alpha$ values on the Catphan and the pelvis patient case.

material sparsity. This is reflected in the best quantitative performance achieved with this norm. However, due to its extreme nonconvexity, it is more challenging to tune the parameters for convergence to an acceptable local minimum. The sparsity terms with $\alpha=\frac{1}{2}$ and $\alpha=\frac{2}{3}$ are better behaving nonconvex functions, showing more robust performance to optimization parameter selection.

The nonconvex optimization problem in this study was solved using an accelerated primal-dual algorithm with line search proposed in Ref. 12. The primal-dual algorithm reduces 
to the primal-dual hybrid gradient (PDHG) method ${ }^{16,17}$ when applied to convex optimization problems. PDHG is one of the first-ordered algorithms that are well-suited for large-scaled optimization problems. Other first-ordered algorithms such as the fast iterative shrinkage-thresholding algorithm, ${ }^{18}$ which achieves a convergence rate of $O\left(\frac{1}{k^{2}}\right)$, substantially faster than the $O\left(\frac{1}{k}\right)$ convergence rate of the PDHG, will be investigated in future studies on the nonconvex optimization problems.

\section{Conclusions}

The proposed method accurately decomposed the DECT phantom and patient images up to 12 basis materials, markedly reduced cross talk among materials, suppressed decomposition image noise, and retained image spatial resolution. The proposed method using nonconvex material sparsity penalty outperforms convex penalty and the standard DI method.

\section{Appendix}

\subsection{Evaluate the Proximal Operator of $\eta|\cdot|^{\alpha}$ for Different $\alpha$ Values}

For $\alpha=0$, the proximal operator in Eq. (5) reduces to a hard thresholding operation:

$$
\left(\operatorname{Prox}_{w F_{3}}\left(\hat{z}_{3}\right)\right)_{i}=\left\{\begin{array}{cl}
\hat{z}_{3 i}, & \text { if }\left|\hat{z}_{3 i}\right| \geq \sqrt{2 \eta w} \\
0, & \text { if }\left|\hat{z}_{3 i}\right|<\sqrt{2 \eta w}
\end{array}\right.
$$

For $\alpha=\frac{1}{2}$, the closed-form solution was proposed by McKelvey: ${ }^{19}$

$$
\begin{aligned}
& \left(\operatorname{Prox}_{w F_{3}}\left(\hat{z}_{3}\right)\right)_{i} \\
& \quad= \begin{cases}\frac{4}{3} \sin ^{2}\left(\frac{1}{3} \arccos \left(\frac{3 \sqrt{3}}{4} u\right)+\frac{\pi}{2}\right), & \text { if } u \leq \frac{2 \sqrt{6}}{9} \\
0, & \text { if } u>\frac{2 \sqrt{6}}{9}\end{cases}
\end{aligned}
$$

where $u=\eta w\left|\hat{z}_{3 i}\right|^{-\frac{3}{2}}$.

For $\alpha=\frac{2}{3}$, the solution of the proximal operator is a root of the quartic polynomial:

$x^{4}-3 \hat{z}_{3 i} x^{3}+3 \hat{z}_{3 i}^{2} x^{2}-\hat{z}_{3 i}^{3} x+\frac{8 \eta^{3} w^{3}}{27}=0$,

for which the analytic solution exists and could be evaluated following the work in Ref. 20. The details on the proximal operator evaluation for $\alpha=\frac{2}{3}$ are shown in Algorithm 3 .

For $\alpha=1$, the proximal operator results in soft thresholding:

$$
\left(\operatorname{Prox}_{w F_{3}}\left(\hat{z}_{3}\right)\right)_{i}= \begin{cases}\hat{z}_{3 i}-\eta, & \text { if } \hat{z}_{3 i} \geq \eta, \\ 0, & \text { if }\left|\hat{z}_{3 i}\right|<\eta, . \\ \hat{z}_{3 i}+\eta, & \text { if } \hat{z}_{3 i} \leq-\eta\end{cases}
$$

\section{$6.2 L A C$ Values}

Table 4 presents the HE LAC and LE LAC values of the basis materials, which were computed as the average values of the $\mathrm{HE}$ LACs and LE LACs within the ROIs shown on the LE CT images in Figs. 1, 3, 5, and 7.
Algorithm 3 Proximal operator evaluation ( $\alpha=2 / 3)$, the solution to Eqs. (5) and (7).

Compute intermediary terms $\boldsymbol{a}_{1}, \boldsymbol{a}_{2}, \boldsymbol{a}_{3}, \boldsymbol{a}_{4}$ :

$$
\begin{aligned}
\boldsymbol{a}_{1} & :=\frac{8 w^{3} \eta^{3}}{27 \hat{z}_{3 i}^{4}}, \quad \boldsymbol{a}_{2}:=\sqrt[3]{\frac{\boldsymbol{a}_{1}}{16}+\sqrt{-\frac{\boldsymbol{a}_{1}^{3}}{27}+\frac{\boldsymbol{a}_{1}^{2}}{256}}}, \quad \mathbf{a}_{3}:=\frac{5}{8}+2 \boldsymbol{a}_{2}+\frac{2 \boldsymbol{a}_{1}}{3 \boldsymbol{a}_{2}}, \\
\boldsymbol{a}_{4} & :=\sqrt{\boldsymbol{a}_{3}-\frac{3}{8}}
\end{aligned}
$$

Compute 4 roots $\boldsymbol{r}_{1}, \boldsymbol{r}_{2}, \boldsymbol{r}_{3}, \boldsymbol{r}_{4}$ :

$$
\begin{gathered}
r_{1}:=\frac{3}{4}+\left(a_{4}+\sqrt{\frac{9}{8}-a_{3}-\frac{1}{4 a_{4}}}\right) / 2, \\
r_{2}:=\frac{3}{4}+\left(a_{4}-\sqrt{\frac{9}{8}-a_{3}-\frac{1}{4 a_{4}}}\right) / 2 \\
r_{3}:=\frac{3}{4}+\left(-a_{4}+\sqrt{\frac{9}{8}-a_{3}+\frac{1}{4 a_{4}}}\right) / 2, \\
r_{4}:=\frac{3}{4}+\left(-a_{4}-\sqrt{\frac{9}{8}-a_{3}+\frac{1}{4 a_{4}}}\right) / 2
\end{gathered}
$$

\begin{tabular}{|c|c|c|c|c|c|c|c|c|}
\hline \multirow[b]{2}{*}{$\begin{array}{l}\mathrm{ROI} \\
\#\end{array}$} & \multicolumn{2}{|c|}{$\begin{array}{c}\text { Digital } \\
\text { phantom }\end{array}$} & \multicolumn{2}{|c|}{ Catphan } & \multicolumn{2}{|c|}{$\begin{array}{l}\text { Quantitative } \\
\text { imaging } \\
\text { phantom }\end{array}$} & \multicolumn{2}{|c|}{ Pelvis patient } \\
\hline & $\begin{array}{c}\mathrm{LE} \\
\left(\mathrm{mm}^{-1}\right)\end{array}$ & $\underset{\left(\mathrm{mm}^{-1}\right)}{\mathrm{HE}}$ & $\begin{array}{c}\mathrm{LE} \\
\left(\mathrm{mm}^{-1}\right)\end{array}$ & $\underset{\left(\mathrm{mm}^{-1}\right)}{\mathrm{HE}}$ & $\begin{array}{c}\mathrm{LE} \\
\left(\mathrm{mm}^{-1}\right)\end{array}$ & $\begin{array}{c}\mathrm{HE} \\
\left(\mathrm{mm}^{-1}\right)\end{array}$ & $\begin{array}{c}\mathrm{LE} \\
\left(\mathrm{mm}^{-1}\right)\end{array}$ & $\begin{array}{c}\mathrm{HE} \\
\left(\mathrm{mm}^{-1}\right)\end{array}$ \\
\hline 1 & 0.059 & 0.033 & 0.043 & 0.038 & 0.024 & 0.019 & 0.059 & 0.035 \\
\hline 2 & 0.045 & 0.022 & 0.029 & 0.027 & 0.026 & 0.020 & 0.031 & 0.021 \\
\hline 3 & 0.023 & 0.017 & 0.033 & 0.027 & 0.029 & 0.021 & 0.025 & 0.019 \\
\hline 4 & 0.001 & 0.000 & 0.018 & 0.017 & 0.031 & 0.022 & 0.020 & 0.016 \\
\hline 5 & & & 0.024 & 0.022 & 0.027 & 0.022 & 0.000 & 0.000 \\
\hline 6 & & & 0.003 & 0.003 & 0.030 & 0.024 & & \\
\hline 7 & & & & & 0.043 & 0.030 & & \\
\hline 8 & & & & & 0.025 & 0.020 & & \\
\hline 9 & & & & & 0.025 & 0.021 & & \\
\hline 10 & & & & & 0.021 & 0.018 & & \\
\hline 11 & & & & & 0.023 & 0.019 & & \\
\hline 12 & & & & & 0.023 & 0.019 & & \\
\hline 13 & & & & & 0.000 & 0.000 & & \\
\hline
\end{tabular}

Pick the root $\boldsymbol{r}$ as the maximum of $\left\{0, \boldsymbol{r}_{1}, \boldsymbol{r}_{2}, \boldsymbol{r}_{3}, \boldsymbol{r}_{4}\right\}$ that satisfies

1. $r$ is a real number

2. $0.5<r<1$

Output: $\left(\operatorname{Prox}_{w F_{3}}\left(\hat{z}_{3}\right)\right)_{i}=r \hat{z}_{3 i}$

Table 4 The HE LAC and LE LAC values of the basis materials in all cases. 


\section{Disclosures}

No conflicts of interest, financial or otherwise, are declared by the authors.

\section{Acknowledgments}

This research is supported by DOE under Grant Nos. DESC0017057 and DE-SC0017687, and NIH under Grant Nos. R44CA183390, R01CA188300, R01CA230278, R21CA228160, and R43CA183390. We would like to thank Shuai Leng at Mayo Clinic for providing the quantitative imaging phantom DECT data. An earlier version of this work was published in the conference proceedings of SPIE Medical Imaging 2019. ${ }^{21}$

\section{References}

1. M. D. Agrawal et al., "Oncologic applications of dual-energy CT in the abdomen," RadioGraphics 34(3), 589-612 (2014).

2. H. A. Lee et al., "Comparison of virtual unenhanced images derived from dual-energy CT with true unenhanced images in evaluation of gallstone disease," Am. J. Roentgenol. 206(1), 74-80 (2016).

3. Q. Wang et al., "Quantitative analysis of the dual-energy CT virtual spectral curve for focal liver lesions characterization," Eur. J. Radiol. 83(10), 1759-1764 (2014).

4. D. T. Boll et al., "Renal stone assessment with dual-energy multidetector CT and advanced postprocessing techniques: improved characterization of renal stone composition-pilot study," Radiology 250(3), 813-820 (2009)

5. C. N. De Cecco et al., "Dual-energy CT: oncologic applications," Am. J. Roentgenol. 199(Suppl. 5), S98-S105 (2012).

6. R. E. Alvarez and A. MacOvski, "Energy-selective reconstructions in x-ray computerized tomography," Phys. Med. Biol. 21, 733-744 (1976).

7. A. Macovski et al., "Energy dependent reconstruction in x-ray computerized tomography," Comput. Biol. Med. 6, 325-334, IN7, 335-336 (1976).

8. C. H. McCollough et al., "Dual- and multi-energy CT: principles, technical approaches, and clinical applications," Radiology 276(3), 637-653 (2015).

9. Y. Long and J. A. Fessler, "Multi-material decomposition using statistical image reconstruction for spectral CT," IEEE Trans. Med. Imaging 33(8), 1614-1626 (2014).

10. P. R. S. Mendonça, P. Lamb, and D. V. Sahani, "A flexible method for multi-material decomposition of dual-energy CT images," IEEE Trans. Med. Imaging 33(1), 99-116 (2014).

11. T. Moellenhoff et al., "Low rank priors for color image regularization," Lect. Notes Comput. Sci. 8932, 126-140 (2014).

12. Y. Malitsky and T. Pock, "A first-order primal-dual algorithm with linesearch," SIAM J. Optim. 28(1), 411-432 (2018).
13. N. Parikh and S. Boyd, "Proximal algorithms," Found. Trends Optim. 1(3), 123-231 (2013).

14. W. Wang and M.-Á. Carreira-Perpiñán, "Projection onto the probability simplex: an efficient algorithm with a simple proof, and an application," arXiv:1309.1541 (2013)

15. S. Boyd and L. Vandenberghe, "Convex optimization," Cambridge University Press, Cambridge (2004).

16. T. Pock et al., "An algorithm for minimizing the Mumford-Shah functional," in Proc. IEEE Int. Conf. Comput. Vision (2009).

17. A. Chambolle and T. Pock, "A first-order primal-dual algorithm for convex problems with applications to imaging," J. Math. Imaging Vision 40(1), 120-145 (2011).

18. A. Beck and M. Teboulle, "A fast iterative shrinkage-thresholding algorithm for linear inverse problems," SIAM J. Imaging Sci. 2(1), 183-202 (2009).

19. J. P. McKelvey, "Simple transcendental expressions for the roots of cubic equations," Am. J. Phys. 52(3), 269-270 (1984).

20. D. Krishnan and R. Fergus, "Fast image deconvolution using hyperLaplacian priors," in Proc. 22nd Int. Conf. Neural Inf. Process. Syst., Y. Bengio et al., Eds., Curran Associates, Inc., pp. 1033-1041 (2009).

21. Q. Lyu et al., "Image-domain multi-material decomposition for dualenergy CT with non-convex sparsity regularization," Proc. SPIE 10949, 1094903 (2019).

Qihui Lyu received her BS degree in physics from Nanjing University in Nanjing, China. Currently, she is a PhD candidate in the Physics and Biology in Medicine Program at University of California, Los Angeles. She is interested in advanced optimization algorithms for inverse treatment planning, medical image reconstruction, and dual-energy CT.

Daniel O'Connor received his $\mathrm{PhD}$ in mathematics from University of California, Los Angeles. $\mathrm{He}$ is an assistant professor at University of San Francisco. His postdoctoral research in the UCLA Department of Radiation Oncology focused on applications of optimization in radiation therapy, medical imaging, and machine learning. His research interests are in large scale optimization modeling and algorithms.

Tianye Niu received his $\mathrm{PhD}$ in physics and electronics from the University of Science and Technology of China. He is a professor at the Institute of Translational Medicine at Zhejiang University. His research interests include compressed sensing, low-dose reconstruction, dual-energy CT imaging, spectral CT imaging, IGRT, proton therapy, radiomics, and cone-beam CT instrument.

Ke Sheng received his $\mathrm{PhD}$ in medical physics from the University of Wisconsin-Madison. He is a professor and an associate vice chair of radiation oncology at University of California, Los Angeles. His main research areas include inverse optimization, $4 \pi$ noncoplanar radiotherapy, image guided radiotherapy, biological outcome modeling, and small animal irradiation. 